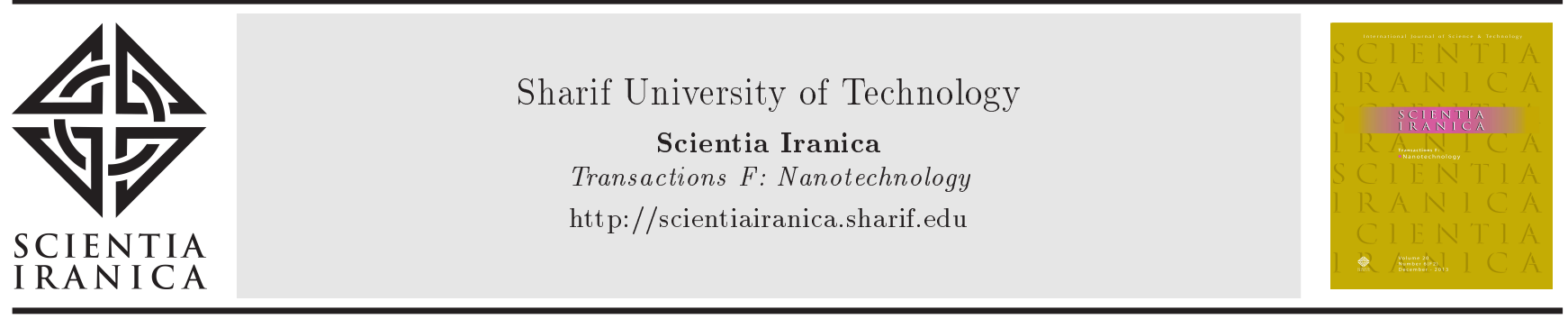

\title{
Electrical conductivity of doped armchair graphene nanoribbon in the presence of gap parameter
}

\author{
H. Rezania*, S. Goli, and A. Jazideh \\ Department of Physics, Razi University, Kermanshah, P.O. Box 0786534239456, Iran.
}

Received 22 November 2016; received in revised form 17 May 2017; accepted 13 January 2018

\section{KEYWORDS}

A. Armchair;

D. nanoribbon;

D. Green's function.

\begin{abstract}
We address the electronic properties of armchair graphene nanoribbon with intight binding model Hamiltonian. Specifically, we have investigated the behavior of density of states and electrical conductivity. The possible gap parameter effects, ribbon width, and chemical potential on electrical conductivity are investigated. Using Green's function, the electrical conductivity and density of states of the system have been calculated. Based on the results, the band gap in density of states increases with gap parameter and decreases with ribbon width. The dependence of the electrical conductivity on temperature for various ribbon widths and chemical potentials has been found. Our results show that a peak appears in temperature dependence of electrical conductivity for each value of chemical potential and ribbon width.
\end{abstract}

(C) 2018 Sharif University of Technology. All rights reserved.

\section{Introduction}

Recently, a single sheet of graphite (Graphene), has drawn the attention of both theoretical and experimental condensed matter physicists due to its novel electronic and thermal properties [1-3]. The novel physical properties come from the fact that the excitations are massless Dirac fermions, which could lead to physical behavior different from that of the standard two-dimensional systems $[1,4,5]$. One can mention that a number of anomalous phenomena ranging from half integer quantum Hall effect, nonzero Berry's phase [6], to minimum conductivity [1] have been observed in experiments. Due to its high values for mobility [7]

*. Corresponding author. Tel./Fax: +988314274569 E-mail addresses: rezania.hamed@gmail.com (H. Rezania); s.goli@gmail.com (S. Goli); a.jazideh@gmail.com (A. Jazideh)

doi: $10.24200 /$ sci.2018.20106 and electrical conductivity [3], graphene is recognized as one of the key materials for realizing electronic devices of the next generation. The presence of edges in graphene has strong implications for the low-energy spectrum of the $\pi$-electrons [8-10]. The properties of graphene ribbons are determined through two basic edge shapes, namely, armchair and zigzag.

The construction of graphene nanoribbons with various widths is possible with using the mechanical method [6] and the epitaxial growth [11] method. The high degree of roughness at the edges can significantly change the properties of edge states [12]. Such edge disorder leads to Anderson localization and Coulomb blockade effects [13]. The edge passivation can be modeled through the tight binding approach by modifications of hopping energies [14] or via additional phases in the boundary conditions [15]. Spin dependent transport properties such as spin hall effect of graphene nanoribbons have recently been calculated [16]. Ribbons with zigzag edges possess localized edge states with energies close to the Fermi level $[9,17]$. Optical 
selection rules of zigzag graphene nanoribbons have been presented in a theoretical work $[18,19]$. On the other hand, edge states are absent from ribbons with armchair edges. A number of research groups [20,21] have derived the analytical wave function and energy dispersion of zigzag nanoribbon. Using the effective-mass approximation [22-24], the analytical forms of electronic wave functions of armchair graphene nanoribbons within the low-energy range have been found. Based on the achieved, all zigzag graphene nanoribbons are metallic with localized states on the edges [20,21] while armchair graphene nanoribbons are either metallic or insulating, depending on their widths $[22,20]$. Moreover the authors of a theoretical paper have derived an analytical expression for electronic band energy and energy spectrum in armchair graphene nanoribbons [25]. Because of quantum confinement effects, the spectrum breaks into a set of subbands and the wave vector along the confined direction becomes discretized [26]. The study of properties of armchair graphene nanoribbons is an interesting topic due to achievements in fabrication of narrow nanoribbons characterized by well-controlled width $[27,28]$. The dynamical and static spin susceptibilities of graphene nanoribbon have been studied using random phase approximation [29]. Analytical study of electronic properties of gapped graphene nanoribbons has been performed [30].

We investigate the effects of ribbon width on temperature dependence of electrical conductivity of armchair graphene nanoribbon by using tight binding model Hamiltonian. The static electrical conductivity has been obtained using Green's function method. By means of the electronic band structure, the Green's function matrix elements have readily been found. In the last section, we will discuss and analyze our numerical results regarding the effects of ribbon width and chemical potential on the dependence of the electrical conductivity on the temperature. Moreover, we investigate the impact of gap parameter on the density of states and electrical conductivity of armchair graphene nanoribbon.

\section{Theoretical formalism}

The electronic structure of gapped graphene nanoribbon can be obtained in the context of a nearest neighbor tight-binding model for $\pi$ electrons. This model Hamiltonian is introduced by:

$$
H=-t \sum_{\left\langle m m^{\prime}\right\rangle}\left(c_{m}^{\dagger} c_{m^{\prime}}+\text { h.c. }\right)-\sum_{m}\left(\epsilon_{m} c_{m}^{\dagger} c_{m}\right),
$$

in which $\left\langle m m^{\prime}\right\rangle$ implies the nearest neighbor lattice sites; h.c. denotes the Hermitian conjugate; operator $c_{m}^{\dagger}\left(c_{m}\right)$ creates (annihilates) an electron at the $m$ th site of the lattice; $\epsilon_{m}$ denotes the on-site energy; $t$ implies the hopping integral of electrons from one atomic site to other one. Since no long-range magnetic ordering has been assumed for electrons, the spin index of electrons does not require to be considered. Figure 1 shows crystal structure of armchair graphene nanoribbon lattice consisting of two types of sublattices $A$ and $B$. The unit cell contains $n A$-type atoms and $n B$ type atoms. Translational invariance along $x$ direction results in defining the following electronic creation $c_{A_{i}\left(B_{i}\right), k_{x}}^{\dagger}$ operator using Fourier transformation:

$$
\begin{aligned}
& c_{A_{i}, k_{x}}^{\dagger}=\frac{1}{\sqrt{N}} \sum_{l=1}^{N} e^{i k_{x} x_{l}} c_{A_{i}, l}^{\dagger} \\
& c_{B_{i}, k_{x}}^{\dagger}=\frac{1}{\sqrt{N}} \sum_{l=1}^{N} e^{i k_{x} x_{l}} c_{B_{i}, l}^{\dagger},
\end{aligned}
$$

where $c_{A_{i}\left(B_{i}\right), k_{x}}^{\dagger}$ creates an electron with wave number $k_{x}$ at $A(B)$ sublattice with position $i$ along the width of the ribbon, i.e. $y$ direction as shown in Figure 1. $x_{l}$ refers to the position of the $l$ th unit cell of nanoribbon lattice along $x$ direction according to Figure $1 . N$ is the number of unit cells along $x$ direction. $k_{x}$ is a wave vector in the first Brillouin zone of nanoribbon lattice of which the constant is $3 a$, according to Figure 1 . $c_{A_{i}, l}^{\dagger}\left(c_{B_{i}, l}^{\dagger}\right)$ creates an electron in $p_{z}$ orbit of a carbon atom located at $A(B)$ sublattices at position $i$ along the width of ribbon, i.e. $y$ direction, on the $l$ th unit cell. The band spectrum of electrons in armchair graphene nanoribbon is obtained by considering the following expansions for the wave functions of $A$ and $B$ sublattices in operator form $\left(c_{A, k_{x}, p}^{\dagger}, c_{B, k_{x}, p}^{\dagger}\right)[25]$ :

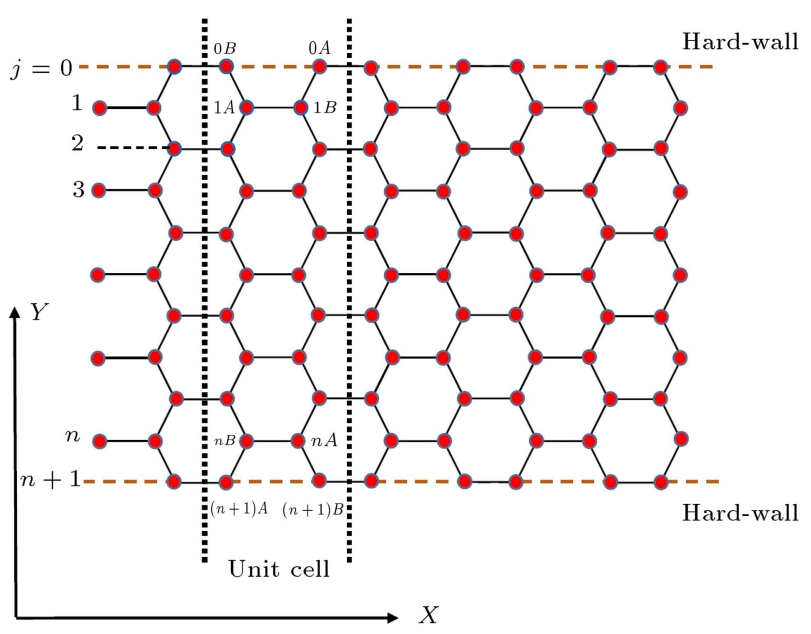

Figure 1. Structure of an armchair graphene nanoribbon, consisting of sublattices, $A$ and $B . n$ is the width of the ribbon. Every unit cell contains a number, $n$, of atoms of the $A$ and $B$ sublattices. Two additional hard walls $(j=0, n+1)$ are imposed on both edges. 


$$
\begin{aligned}
c_{A, k_{x}, p}^{\dagger} & =\sqrt{\frac{2}{(n+1)} \sum_{i=1}^{n} \sin \left(\frac{p \pi}{n+1} i\right) c_{A_{i}, k_{x}}^{\dagger},} \\
c_{B, k_{x}, p}^{\dagger} & =\sqrt{\frac{2}{(n+1)} \sum_{i=1}^{n} \sin \left(\frac{p \pi}{n+1} i\right) c_{B_{i}, k_{x}}^{\dagger} .}
\end{aligned}
$$

so that $c_{A(B), k_{x}, p}^{\dagger}$ creates an electron at sublattice $A(B)$ with wave vector $k_{x}$ belonging to the first Brillouin zone. $n$ denotes the width of ribbon and $p$ is an integer number with values $p=1,2,3, \ldots, n$. Hard-wall boundary condition [25] implies the factor $\sin \left(\frac{p \pi}{n+1} i\right)$ in the expansion in Eq. (3). The tight binding model Hamiltonian is written in terms of basis kets $\left\{\left|\psi_{A, k_{x}, p}\right\rangle,\left|\psi_{B, k_{x}, p}\right\rangle\right\}$ as:

$$
\begin{aligned}
H= & \sum_{k_{x}, p}\left(f\left(k_{x}, p\right) c_{A, k_{x}, p}^{\dagger} c_{B, k_{x}, p}+H . c .\right) \\
& -\sum_{k_{x}, p}\left(\epsilon_{A} c_{A, k_{x}, p}^{\dagger} c_{A, k_{x}, p}+\epsilon_{B} c_{B, k_{x}, p}^{\dagger} c_{B, k_{x}, p}\right),
\end{aligned}
$$

where $f\left(k_{x}, p\right)=-t\left[2 e^{i k_{x} a / 2} \cos \left(\frac{p \pi}{n+1}\right)+e^{-i k_{x} a}\right]$. Also, $\epsilon_{A(B)}$ refers to electronic on-site energy of sublattice $A(B)$. Diagonalization of the Hamiltonian in Eq. (4) yields a two-band spectrum. By assuming $\epsilon_{A}=-\epsilon_{B} \equiv$ $\Delta$, the band energies are :

$$
E_{ \pm}\left(k_{x}, p\right)= \pm \sqrt{\left|f\left(k_{x}, p\right)\right|^{2}+\Delta^{2}}
$$

where \pm implies the conduction and valence bands, respectively. Moreover, $\Delta$ introduces the gap parameter. Using band energy spectrum in Eq. (5), the Hamiltonian in Eq. (4) is expressed by:

$$
H=\sum_{k_{x}, p, \eta= \pm} E_{\eta}\left(k_{x}, p\right) c_{\eta, k_{x}, p}^{\dagger} c_{\eta, k_{x}, p} .
$$

The definition of matrix elements for the electronic Matsubara Green's function [31] is given by:

$$
G_{\eta, p}\left(k_{x}, \tau\right)=-\left\langle T_{\tau} c_{\eta, p, k_{x}}(\tau) c_{\eta, p, k_{x}}^{\dagger}(0)\right\rangle
$$

where $\tau$ refers to imaginary time. The Fourier transformation of each matrix element of Green's function is obtained by:

$$
G_{\eta, p}\left(k_{x}, i \omega_{n}\right)=\int_{0}^{1 / k_{B} T} d \tau e^{i \omega_{n} \tau} G_{\eta, p}\left(k_{x}, \tau\right),
$$

and $\omega_{n}=(2 n+1) \pi k_{B} T$ is the fermionic Matsubara frequency in which $T$ is equilibrium temperature.

Using the model Hamiltonian in Eq. (6), we can find the Fourier transformation of each matrix element of Green's function as:

$$
G_{\eta, p}\left(k_{x}, i \omega_{n}\right)=\frac{1}{i \omega_{n}-E_{\eta}\left(k_{x}, p\right)+\mu},
$$

where chemical potential, $\mu_{\sigma}$, is determined by the electronic concentration $\left(n_{e}\right)$ through the following expression:

$$
n_{e}=\frac{1}{n N} \sum_{k_{x}, p, \eta= \pm} \frac{1}{e^{\left(E_{\eta}\left(k_{x}, p\right)-\mu\right) / k_{B} T}+1} .
$$

The amount of electronic concentration $n_{e}$ determines the chemical potential, $\mu$, by means of Eq. (10).

In order to obtain electrical conductivity, the correlation function of the electrical current operators should be calculated. The final result for the singleband model Hamiltonian [32] has been obtained and one can generalize this result to multiband model Hamiltonians. Using the Green's function in band space, the static electrical conductivity of armchair graphene nanoribbon is expressed as follows:

$$
\begin{aligned}
\sigma_{x x}(T)= & \frac{e^{2}}{4 N n} \sum_{k_{x}, p, \eta}\left(\frac{\partial E_{\eta}\left(k_{x}, p\right)}{\partial k_{x}}\right)^{2} \\
& \int_{-\infty}^{\infty} \frac{d \epsilon}{2 \pi}\left(\frac{-\partial n_{F}(\epsilon)}{\partial \epsilon}\right)\left(2 \operatorname { I m } G _ { \eta , p } \left(k_{x}, i \omega_{n}\right.\right. \\
& \left.\left.\left.\longrightarrow \epsilon+i 0^{+}\right)\right)^{2}\right)
\end{aligned}
$$

where $n_{F}(x)=\frac{1}{e^{x / k_{B} T}+1}$ introduces the Fermi-Dirac distribution function. Substituting electronic Green's function into Eq. (11) and integrating over wave vector through interval $-\pi / 3 a<k_{x}<\pi / 3 a$, the results for static electrical conductivity have been obtained. Moreover, density of states is found based on Green's function as:

$$
D(E)=\frac{1}{2 n N} \sum_{k_{x}, p, \eta}-2 \operatorname{Im} G_{\eta, p}\left(k_{x}, i \omega_{n} \longrightarrow E+i 0^{+}\right) .
$$

\section{Numerical results and discussion}

The main numerical results for total density of states and electrical conductivity of armchair graphene nanoribbon in the presence of the effects of electronic concentration, gap parameter, and ribbon width are predicted in this section. The results for electronic density of states and electrical conductivity are obtained by substituting the Green's function matrix elements into Eqs. (11) and (12).

The density of states, $D(E)$, of armchair graphene nanoribbon as a function of energy for different values of ribbon width, namely, $n=3,4,5,7,9,10$, is illustrated in Figure 2. This figure implies two features. The increase in ribbon width, $n$, leads to a decrease in band gap width in density of states. Since the armchair nanoribbon with the mentioned widths is the band insulator, we expect the semimetallic properties of the 


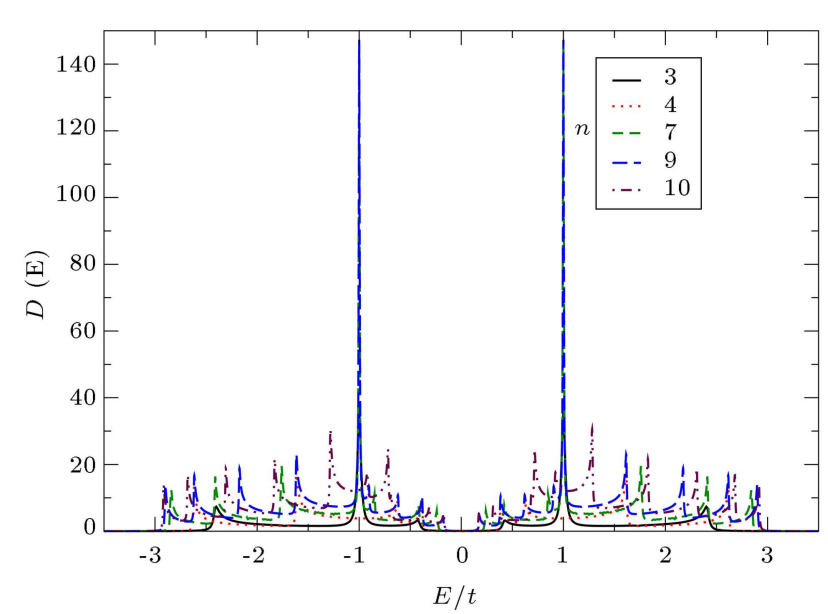

Figure 2. The total density of states $D(E)$ of armchair graphene nanoribbon as a function of the normalized energy, $E / t$, for different ribbon widths, $n$, at $\Delta / t=0$.

graphene sheet to appear by increasing $n$. Therefore the band gap width in the density of states decreases with ribbon width. Moreover, the height of Van Hove singularity increases with $n$ as shown in Figure 2. Also, the difference between the maximum and the minimum energy for which the density of states has a nonzero value is independent of ribbon width $n$. In Figure 3, we show the density of states of armchair graphene nanoribbon with $n=7$ for different values of gap parameter, $\Delta / t$. The increase in on-site energy difference enhances gap parameter, $\Delta$, which in turn increases the band gap in density of states. Since the area below density of states curve corresponds to electronic concentration, this quantity remains constant under the variation of $\Delta / t$ according to Figure 3 . Therefore, the width of density of states increases with gap parameter. We plot the static electrical conductivity of armchair graphene nanoribbon without gap parameter,

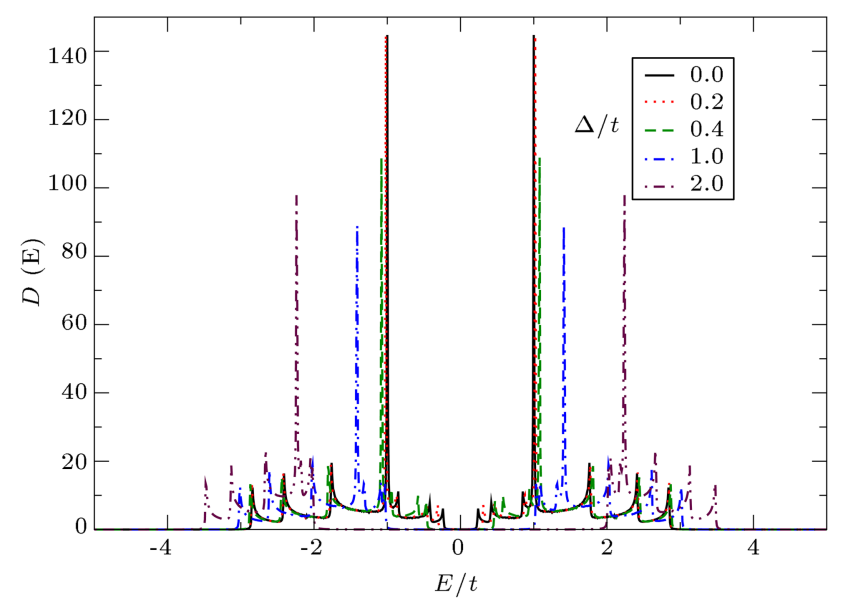

Figure 3. The total density of states $D(E)$ of armchair graphene nanoribbon as a function of the normalized energy, $E / t$, at fixed ribbon width, $n=7$, for different values of gap parameter, $\Delta / t$.

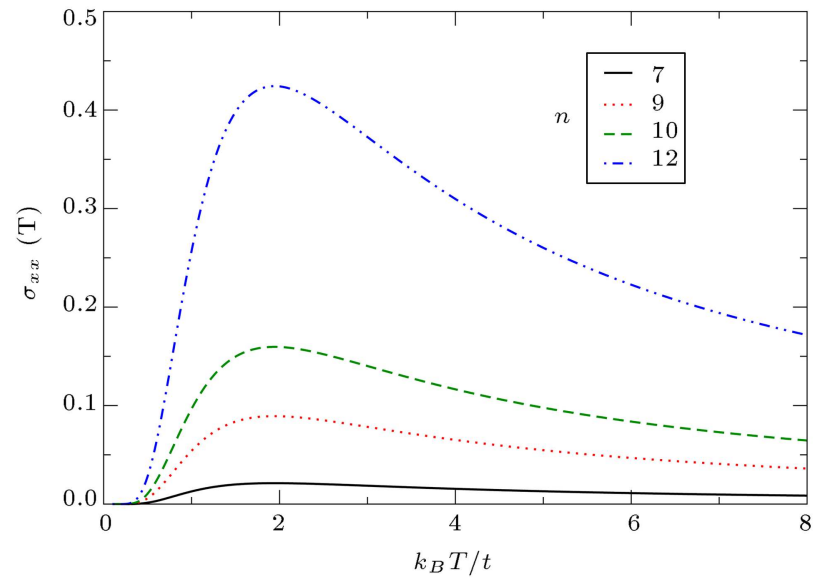

Figure 4. Electrical conductivity of undoped armchair graphene nanoribbon versus normalized temperature, $k_{B} T / t$, for different ribbon widths.

$\Delta / t=0$, versus normalized temperature, $k_{B} T / t$, for different amounts of ribbon width, $n$, in Figure 4. This plot indicates that electrical conductivity reaches the value of zero up to finite nonzero temperature for all values of $n$. Electronic transition from the valence band to the conduction one takes place when temperature reaches a characteristic finite value and, consequently, electrical conductivity begins to increase above this characteristic temperature. Such temperature behavior of electrical conductivity has been predicted in several experimental works [33-35]. Moreover, each curve presents an exponential decay at low temperature, which manifests the presence a finiteenergy gap in excitation spectrum. Lower values of ribbon width lead to more rapid decay corresponding to larger energy gap. There is a peak in the electrical conductivity. The height of peak increases upon increasing $n$. The increasing behavior of $\sigma_{x x}$ can be justified by the point that temperature leads to increase the electronic transition between two bands. However, this is not the case for temperatures above the position of the peak. In this range, the increase in temperature causes more scattering of electrons which reduces the electrical conductivity [36,37]. At a fixed temperature, the electrical conductivity increases with ribbon width, $n$, due to reduction in energy gap. We have also studied the effect of gap parameter, $\Delta$, on temperature behavior of electrical conductivity. In Figure 5, we plot $\sigma_{x x}(T)$ of armchair graphene nanoribbon with $n=$ 7 versus normalized temperature for different values of gap parameter, namely $\Delta / t=0,0.1,0.2,0.3,0.4$. This plot indicates that electrical conductivity reaches the value of zero up to a finite temperature due to energy gap in electronic density of states. Upon more increase temperature, electrical conductivity rises until it reaches a maximum for each gap parameter, $\Delta / t$. The phanomenon can be understood through the fact that temperature enhances the number of electronic 


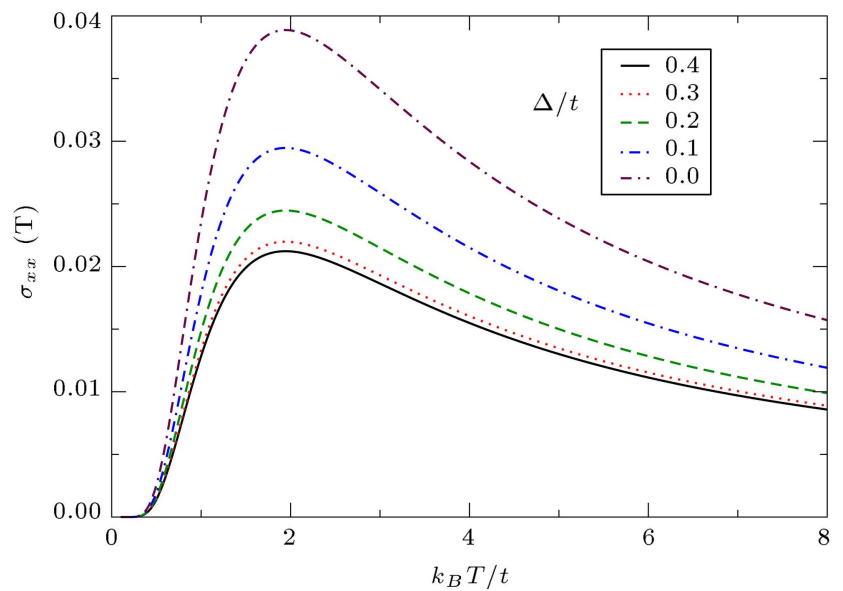

Figure 5. Electrical conductivity of undoped armchair graphene nanoribbon versus normalized temperature, $k_{B} T / t$, for different values of gapped parameter, $\Delta / t$, for $n=7$.

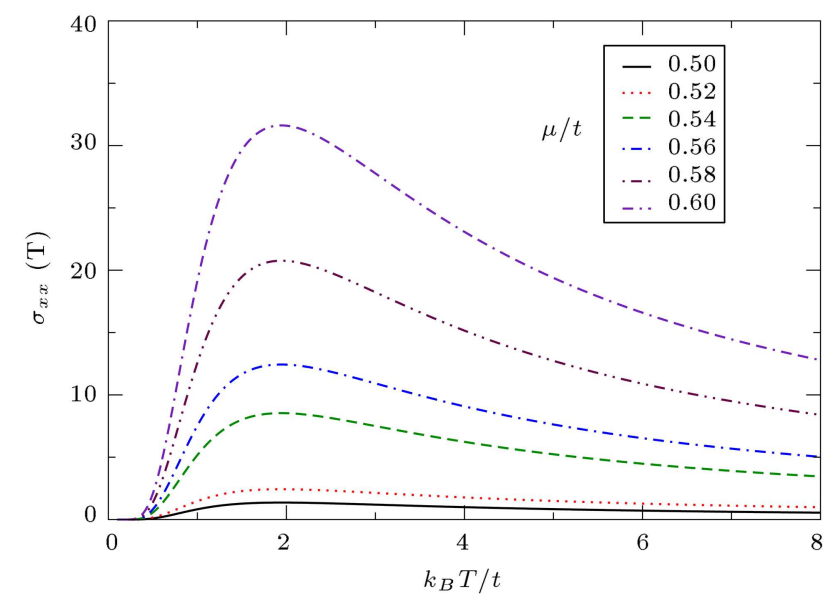

Figure 6. Electrical conductivity of doped armchair graphene nanoribbon versus normalized temperature, $k_{B} T / t$, at fixed ribbon width, $n=7$, for different chemical potential values, $\mu / t$.

transitions from the valence band to the conduction one. Afterwards, electrical conductivity decays for temperatures above peak characteristic temperature. This comes from the effect of scattering of electrons, which reduces the conductivity. In addition, at fixed values of the temperature, lower gap parameter $\Delta / t$ causes less energy gap and, thus, higher values of electrical conductivity. Figure 6 shows the temperature behavior of electrical conductivity of doped armchair graphene nanoribbon with $n=7$ for different chemical potentials. This figure indicates that each curve has a peak at characteristic temperature of $k_{B} T / t=$ 2.0. The other novel feature of this figure is the enhancement of electrical conductivity with increase in chemical potential at fixed temperature. This can be understood from the fact that higher chemical potential yields higher electronic concentration which results in larger electrical conductivity.

\section{Conclusion}

We studied the temperature dependence of electrical conductivity of gapped graphene nanoribbon in the presence of electron doping, which means increase in the concentration of electrons in the system via adding impurity atoms to the structure. Using a tight binding model Hamiltonian and a Green's function approach, the excitation spectrum of the model Hamiltonian was studied. In particular, the effect of gap parameter on electrical conductivity and density of states was investigated. The increase in gap parameter led to decrease in electrical conductivity. Moreover, at a fixed temperature, the increase in ribbon width caused the enhancement of electrical conductivity. On the other hand, our results showed that increase in the chemical potential gave rise to increase in electrical conductivity. The exponential behavior of electrical conductivity of graphene nanoribbon was a sign of energy gap in density of states. In other words at low temperature the conductivity was related to band gap width $(\Delta)$ as $\sigma \propto e^{-\Delta / T}$. Thus, we could read the band gap in density of states by using temperature behavior of static electrical conductivity. One of the most important applications of band gap in density of states is the study of optical absorption spectra of the graphene nanoribbon. The variation of band gap in density of states causes the change of frequency of absorbed electromagnetic wave.

\section{References}

1. Novoselov, K.S., Geim, A.K., Morozov, S.V., Jiang, D., Zhang, Y., Dubons, S.V., Grigorieva, I.V., and Firsov, A.A. "Electric Field in atomically thin carbon films", Science, 306(3), pp. 666-670 (2004).

2. Geim, A.K. and Novoselov, K.S. "The rise of graphene", Nature Mater, 6(2), pp. 183-188 (2007).

3. Balandin, A.A., Ghosh, S., Bao, W., Calizo, I., Teweldebrhan, D., Miao, F., and Lau, C.N. "The electric thermal conductivity of graphene", Nano Lett 8(2), pp. 902-907 (2008).

4. Ohta, T., Bostwick, A., Seyller, T., Horn, K., and Rorenberg, E. "Controlling the electronic structure of bilayer graphene", Science, 313(2), pp. 951-955 (2006).

5. Katsnelson, M.I., Novoselov, K.S., and Geim, A.K. "Chiral tunneling and the Klein paradox in graphene", Nat. Phys, 2(1), pp. 620-925 (2006).

6. Zhang, Y., Tan, T.-W., Stormer, H.L., and Kim, P. "Experimental observation of the quantum Hall effect and Berry phase in graphene", Nature, (London), 438(2), pp. 201-205 (2005). 
7. Bolotin, K.I., Sikes K.J., Jiang, Z., Klima, M., Fudenberg, G., Hone, J., Kim, P., and Stormer, H.L. "Ultrahigh electron mobility in suspended graphene", Solid State Commun, 146(2), pp. 351-356 (2008).

8. Fujita, M., Wakabayashi, K., Nakada, K., and Kusakabe, K. "Peculiar localized state at zigzag graphite edge", J. Phys. Soc. Jpn, 65(2) pp. 1920-1927 (1996).

9. Nakada, K., Fujita, M., Dresselhaus, G., and Dresselhaus, M.S. "Graphene nanoelectronics: Metrology, Synethesis", Phys. Rev. B, 54(2), pp. 17954-17959 (1996).

10. Ezawa, M. "Peculiar phase dependence of the electronic properties of carbon nanoribbons", Phys. Rev. $B, \mathbf{7 3}(3)$, pp. 045432-045438 (2006).

11. Berger, C. "Ultrathin epitaxial graphite: 2D electron gas properties", J. Phys. Chem. B, 108(2), pp. 1991219917 (2004).

12. Areshkin, D.A. and White, C.T. "Ballistic transport in graphene nanostrips in the graphene", Nano Lett, 7(3) pp. 204-208 (2007).

13. Sols, F., Guinea, F., and Castro, Neto, A.H. "Coulomb blockade in graphene nanoribbons", Phys. Rev. Lett, 99(2), pp. 166803-166809 (2007).

14. Novikov, D.S. "Transverse field effect in graphene ribbons", Phys. Rev. Lett, 99(3), pp. 056802-056805 (2007).

15. Kane, C.L. and Male, E.J. "Size, shape and low energy electronic structure of carbon nanotubes", Phys. Rev. Lett, 78(3), pp. 1932-1935 (1997).

16. Van Tuan, D., Marmolejo-Tejada, J.M., Waintal, X., Nikolic, B.K., Valenzuela, S.O., and Roche, S. "Spin hall effect and origins of nonlocal resistance in adatomdecorated graphene", Phys Rev Lett, 117, pp. 176602176605 (2016).

17. Wakabayashi, K., Sigrist, M., and Fujita, M. "Role of edges in the electronic and magnetic structures of nanographene", J. Phys. Soc. Japan, 67(2), pp. 20892095 (1998).

18. Saroka, V.A., Shuba, M.V., and Portnoi, M.E. "Optical selection rules of zigzag graphene nanoribbons", Phys. Rev. B, 95, pp. 155438-155445 (2017).

19. Chung, H.-C., Chang, C.-P., Lin, C.-Y., and Lin, M.-F. "Electronic and optical properties of graphene nanoribbons in external fields", Phys. Chem. Chem. Phys, 18, pp. 7573-7580 (2016).

20. Sasaki, K., Murakami, S., and Saito, R. "Gauge field for edge state in graphene", J. Phys. Soc. Jpn, 75(3), pp. 074713-074720 (2006).

21. Sasaki, K., Murakami, S., and Saito, R. "Stabilization mechanism of edge state in graphene", Appl. Phys. Lett, 88(3), pp. 113110-113117 (2006).

22. Brey, L., Fertig, H.A., and Das Sarma, S. "Dilute graphene antiferromagnet", Phys. Rev. Lett, 99(2), pp. 116802-116805 (2007).

23. Marconcini, P. and Macucci, M. "The k.p method and its application to graphene, carbon nanotubes and graphene nanoribbons: the Dirac equation", La Rivista del Nuovo Cimento, 34(8-9), pp. 489-584 (2011).

24. Brey, L. and Fertig, H.A. "Electronic states of graphene nanoribbons studied with the Dirac equation", Phys. Rev. B, 73(2), pp. 235411-235417 (2006).

25. Zheng, H., Wang, Z.F., Luo, T., Shi, Q.W., and Chen, J. "Analytical study of electronic structure in armchair graphene nanoribbons", Phys. Rev. B, 75(2), pp. 165414-165420 (2007).

26. Ajiki, H. and Ando, T. "Electronic states of carbon nanotubes", J. Phys. Soc. Jpn, 62, pp. 1255-1260 (1993).

27. Blankenburg, S., Cai, J., Ruffleux, P., Jaafar, R., Passerone, D., Feng, X., Mllen, K., Fasel, R., and Pignedoli, C.A. "Intraribbon heterojunction formation in ultranarrow graphene nanoribbons", ACS Nano, 6(3), pp. 2020-2028 (2012).

28. Ruffleux, P., Cai, J., Pumb, C.N., Patthey, L., Prezzi, D., Ferretti, A., Molinari, E., Feng, X., Mllen, K., Pignedoli, C.A., and Fasel, R. "Electronic structure of atomically precise graphene nanoribbons", Acs Nano, 6(3), pp. 6930-6937 (2012)

29. Rezania, H. and Abdi, A. "Dynamical and static spin susceptibilities of doped gapped graphene nanoribbon due to local electronic interaction", Plasmonics, 13(3), pp. 845-854 (2018).

30. Wakabayashi, K., Sasaki, K.-H., Nakanishi, T., and Enoki, T. "Electronic states of gapped nanoribbons and analytical solutions", Science and Technology of Advanced Materials, 11(5), pp. 1-18 (2010).

31. Mahan, G.D., In Many Particle Physics, 3rd Ed., pp. 295-331, Plenum Press, New York (1993).

32. Paul, I. and Kotliar, G. "Thermal transport for many body tight binding models", Phys. Rev. B, 67(2), p. 115131 (2003).

33. Deng, H.-Y., Wakabayashi, K., and Lam, C.-H. "Mode-Matching approach to current blocking effect in graphene nanoribbons", J. Phys. Soc. Japan, 82, pp. 104707-104717 (2013).

34. Deng, H.-Y. and Wakabayashi. K. "Edge effect on a vacancy state in semi-infinite graphene", Phys. Rev. B, 90, pp. 115413-115420 (2014).

35. Luck, J.M. and Avishai, Y. "Unusual electronic properties of clean and disordered zigzag graphene nanoribbons", J. Phys. Condens. Matter, 27, pp. 025301025310 (2015). 
36. Rezania, H. "Electrical conductivity of zigzag carbon nanotubes including Holstein polarons", European Physical Journal B, 85, pp. 1-5 (2012).

37. Mousavi, H. and Bagheri, M. "Effect of Holstein phonons on the electrical conductivity of carbon nanotubes", Physica E, 44, pp. 1722-1724 (2012).

\section{Biographies}

Hamed Rezania is Associate Professor in Theoretical Condensed Matter Physics Department of Razi University of Kermanshah in Iran. His research interests include: The study of electronic properties of nanostructure systems and transport properties of spin model Hamiltonian as strongly correlated systems are his interests.

Shabnam Goli is MSc graduated in Theoretical Condensed Matter Physics Department of Razi University of Kermanshah in Iran. Her research interests include the study of electronic and transport properties of nanostructure systems.

Arisa Jazideh is MSc student in Theoretical Condensed Matter Physics Department of Razi University of Kermanshah in Iran. Her research interests include the study of electronic and transport properties of nanostructure systems. 